\title{
A new method for the determination of very small $\Gamma_{\gamma}$ partial widths
}

Giuseppe Cardella ${ }^{1}$, Luis Acosta ${ }^{1,7}$, Lucrezia Auditore ${ }^{1,4}$, Alberto Camaiani ${ }^{8}$, Enrico De Filippo ${ }^{1}$, Saverio De Luca ${ }^{1,4}$, Nicla Gelli ${ }^{8}$, Brunilde Gnoffo ${ }^{1,3}$, Francisco Favela ${ }^{1}$, Bogdan Fornal $^{9}$, Gaetano Lanzalone ${ }^{2,5}$, Silvia Leoni ${ }^{6}$, Concetta Maiolino ${ }^{2}$, Nunzia Simona Martorana $^{2,3}$, Adriana Nannini ${ }^{8}$, Sebastianella Norella ${ }^{1,4}$, Angelo Pagano ${ }^{1}$, Emanuele Vincenzo Pagano ${ }^{2,3}$, Massimo Papa ${ }^{1}$, Sara Pirrone ${ }^{1}$, Giuseppe Politi ${ }^{1,3}$, Francesco Porto ${ }^{2}$, Lucia Quattrocchi ${ }^{4}$, Francesca Rizzo, ${ }^{2,3}$, Paolo Russotto ${ }^{2,3}$, Domenico Santonocito ${ }^{2}$, Antonio Trifirò ${ }^{1,4}$ and Marina Trimarchì ${ }^{1,4}$

${ }^{1}$ INFN-Sez.Catania, Catania, Italy

${ }^{2}$ LNS-INFN, Catania, Italy

${ }^{3}$ Dipartimento di Fisica e Astronomia Università di Catania, Catania, Italy

${ }^{4}$ Dip. di Scienze Mat. e Inf., Scienze Fisiche e Scienze della Terra Università di Messina, Italy

${ }^{5}$ Facoltà di Ingegneria e Architettura, Universit Kore, Enna, Italy

${ }^{6}$ INFN sezione di Milano and Dip. di Fisica Università di Milano, Italy

${ }^{7}$ Instituto de Fsica, Universidad Nacional Autnoma de Mexico, Mexico

${ }^{8}$ INFN sezione di Firenze and Dip. di Fisica Università di Firenze, Italy

${ }^{9}$ Institute of Nuclear Physics, Polish Academy of Sciences, Krakow, Poland

\begin{abstract}
We present a new method for the measurement of very small $\Gamma_{\gamma}$ partial width that is important for the synthesis of elements in astrophysics. The method is based on the simultaneous detection of scattered beam, residual nucleus and decay $\gamma$ rays. This method is optimized for the use of the CHIMERA detector at LNS. Experimental details are described.
\end{abstract}

\section{Introduction}

Gamma ray decay partial widths of nuclear levels, at excitation energy larger than the particle decay energy threshold, are generally quite small. Due to large background, it is difficult to measure them with enough accuracy. However, in some cases they are rather important in order to explain the synthesis of the elements in stellar environments. For instance, only after a $\gamma$-decay a $\mathrm{C}$ nucleus is produced in the triple alpha reaction, passing through the Hoyle state, see for instance [1] and references therein. Therefore, the exact knowledge of its decay gamma width is fundamental in order to explain the " $\mathrm{C}$ abundance in the universe. Many experiments were performed in the $70^{\prime \prime}$ of last century, in order to get reliable values for the $\gamma$-rays decay widths of high energy levels of "C. Two main methods were used: the direct measurements of coincidence $\gamma$-rays (two $\gamma$-rays are emitted in the case of the decay of the 0 Hoyle state through the $4.44 \mathrm{MeV} 2^{+}$level) [2]; or the detection 
of "C residual nuclei in coincidence with inelastically scattered projectile particles [3-5]. This last method was more used due to the small detection efficiency of $\gamma$-ray detectors available at that time. Another measurement has been recently performed mainly to improve the knowledge of the $9.64 \mathrm{MeV}$ gamma decay width [6]. The measurement relied on the very precise detection of residual "C nucleus with the Grand Rayden magnetic spectrometer. In order to decrease background beam has been used (therefore excluding "C contaminants in the target, the limiting factor of ref. [7]) and a solid hydrogen target has been developed.

At LNS Catania, we recently proposed a new method to measure such kind of partial gamma widths by using a multiple fold coincidence technique with the CHIMERA $4 \pi$ detector [8]. The first test experiment will be performed at the beginning of November 2017. The partial gamma width of the "C Hoyle state [9] will be measured in order to confirm the validity of the method. At the same time, we expect also to measure or, at least, to improve the known upper limit of the $\gamma$-decay width of the $9.64 \mathrm{MeV} 3$ level [7]. The population of this level seems in fact important for the understanding of the "C production in very hot astrophysical environments [6]. We will also investigate on the possible population and decay of the 2 level, expected in the same energy region that should belong to the rotational band based on the Hoyle state [10]. This technique can be extended to radioactive nuclei, produced by the LNS fragmentation beam line [11], using inverse kinematic reactions on proton targets [12]. Details of the technique and preliminary results will be shown in the following.

\section{The experimental method}

At LNS Catania, we proposed the following new approach, taking advantage of the opportunities offered by the CHIMERA $4 \pi$ detector. Such array is composed by 1192 telescopes, with a first stage of $300 \mu \mathrm{m}$ thick silicon detectors and a second stage of $\mathrm{CsI}(\mathrm{Tl})$ scintillators, with photodiode readout, with length from 3 to $12 \mathrm{~cm}$ [8]. The new GET full digital electronics [13] is used for the readout of the CsI(Tl) stage, allowing the storage of the preamplifier signals. The shape analysis of the signal is a simple way in order to discriminate particles from $\gamma$-rays and isotopically identify charged particles up to Lithium as described in $[14,15]$. An example of the difference in shape of the signals collected for different particles is given in fig.1. With CHIMERA, we can detect both the residual "C (stopped in the $300 \mu \mathrm{m}$ first stage silicon detectors) and $\gamma$-rays (in the thick, second stage CsI(Tl) scintillator), therefore in this case the high expected random background will be reduced using the cleaning effect of up to four fold time coincidence techniques. The cut of the background relies not only on the pure coincidence time, (quite good, of the order of ns, for silicon detectors, reasonable, of the order of hundreds of ns, for the CsI(Tl) signals) but also on the particle identification quality of the detector, on kinematic properties, and on energy conservation rules. The simple coincidence between " $\mathrm{C}$ and the scattered projectile produces a reduction of random background of the order of 10 , (assuming a rate of the order of $1 \mathrm{kHz}$ ) due to the relatively good typical time resolution of few ns of silicon detectors. A further background reduction, by at least another order of magnitude, is obtained fulfilling the constraints due to energy conservation. The request of kinematical coincidence, due to the opening angle of our detectors, reduces the random coincidences by a factor of the order of 30 [12]; particle identifications, with time of flight, $\Delta \mathrm{E}-\mathrm{E}$, and pulse shape analysis of particles stopped in silicon detectors [16], produce another order of magnitude reduction. 


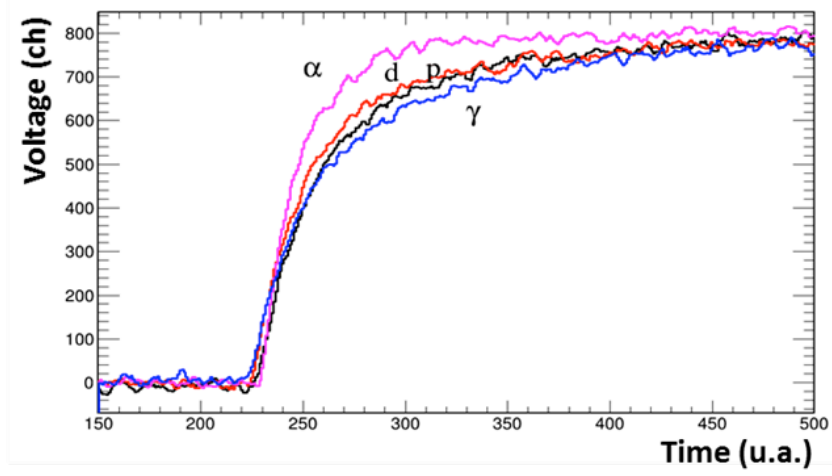

Fig. 1. Examples of signals collected with GET electronics for different particles and $\gamma$-rays.

In total, two-particle coincidences in silicon detectors produce a background suppression of the order of 10" (see table 1).This is not enough (see ref. [7]) to clean from " $\mathrm{C}$ background in the target. However, a further suppression factor can be attained detecting also $\gamma$-rays. Looking to the 9.64 level, because it has a spin 3 and negative parity, we can have both E3 direct decay to the ground state and E1 decay to the $4.44 \mathrm{MeV} 2+$ level, with a subsequent E2 decay to ground state. Looking to the decay strengths the emission of $2 \gamma$-rays of 5.2 and 4.44 $\mathrm{MeV}$ seem the most probable. The coincidence measurement of each $\gamma$-ray, still assuming a rate of the order of one $\mathrm{kHz}$ in the detector, but with the worse time resolution of the CsI(Tl), produces a background suppression factor of at least 3 order of magnitude. Further constraints are given by $\gamma$-rays detected energy, and by particle-gamma discrimination properties in $\mathrm{CsI}(\mathrm{Tl})$. Therefore, the 4-fold coincidence measurement proposed results in a suppression factor of the background of the order of $10^{\text {ss }}$ at least.

In ref. [12] we have already shown the quality of kinematical coincidences measurements performed with CHIMERA detector. Gamma-ray detection efficiency and the possibility to measure $\gamma$-ray angular correlations with CHIMERA was shown in ref. [17]. Angular correlation measurements will be very important to identify the possible decay of a $2+$ level for which evidences near $10 \mathrm{MeV}$ exists [10]. This level is very interesting because it is expected to belong to the presumable rotational band based on the Hoyle state so could be another important brick of the "C production in the universe.

TABLE 1 summary of suppression factors for background with 2,3 and 4 fold coincidences

\begin{tabular}{|c|c|c|c|c|c|c|c|c|c|c|}
\hline & $\begin{array}{c}10 \mathrm{~ns} \\
\text { coincidence } \\
\text { time (si-det) }\end{array}$ & $\begin{array}{l}\text { kinematical } \\
\text { coincidence }\end{array}$ & $\begin{array}{c}\text { energy } \\
\text { conservation }\end{array}$ & $\begin{array}{c}\text { particle } \\
\text { identification }\end{array}$ & $\begin{array}{c}\text { total } 2 \\
\text { particle } \\
\text { coincidence }\end{array}$ & $\begin{array}{c}1 \mu \mathrm{S} \\
\text { coincidence } \\
\text { time(Csl-det) }\end{array}$ & $\begin{array}{c}\text { energy } \\
\text { conservation }\end{array}$ & $\begin{array}{c}\text { particle } \\
\text { identification }\end{array}$ & $\begin{array}{c}\text { total } 3 \text { fold } \\
\text { coincidence }\end{array}$ & $\begin{array}{l}\text { total } 4 \text { fold } \\
\text { coincidence }\end{array}$ \\
\hline $\begin{array}{c}\text { suppression } \\
\text { factor }\end{array}$ & $1,0 \mathrm{E}+05$ & $3,0 \mathrm{E}+01$ & $1,0 \mathrm{E}+01$ & $3,0 \mathrm{E}+00$ & $9,0 \mathrm{E}+07$ & $1,0 \mathrm{E}+03$ & $1,0 \mathrm{E}+01$ & $3,0 \mathrm{E}+00$ & $2,7 \mathrm{E}+12$ & $8,1 \mathrm{E}+15$ \\
\hline
\end{tabular}

The main trigger of the experiment will be the coincidence between projectile and target in silicon detectors. In order to reduce the contribution of the elastic channel we plan to trigger only with detectors belonging to the spherical region of the CHIMERA detector $\left(\theta_{\mathrm{w}}>30^{\circ}\right)$. In this region, we expect to have a relative contribution of elastic and inelastic channels similar to what we have already measured with proton beam, at $24 \mathrm{MeV}$, shown in fig.2. In this figure, we report the Q-value spectrum showing the elastic channel and excited levels. We note that the cross section to populate the resonance at $9.63 \mathrm{MeV}$ is of the same order of magnitude of the $4.44 \mathrm{MeV}$ level, and a factor about 20 larger than the probability to 
populate the Hoyle state at $7.65 \mathrm{MeV}$. This will help to get enough statistics also for the $9.63 \mathrm{MeV}$ notwithstanding the smaller expected $\gamma$-decay probability.

In summary, we are planning to perform a new measurement of the $\gamma$-decay probability of the Hoyle state and of the group of levels around $9.63 \mathrm{MeV}$, including a possible 2+ excited around $10 \mathrm{MeV}$, which should belong to a rotational band based on the Hoyle state. We will use a 4-fold coincidence method decreasing the background of at least 15 orders of magnitude. We expect to collect at least $5 \times 10$ event for the decay of the Hoyle state, i.e. about 2000 coincidences with "C, and around 100 4-fold coincidences with 2 gamma rays. We will get some $10^{*}$ events for the $9.63 \mathrm{MeV}$ level. From which we expect to have up to 100 coincidences $\alpha-{ }^{-2} \mathrm{C}$ and up to 203 -fold and few 4-fold coincidences with gamma rays.

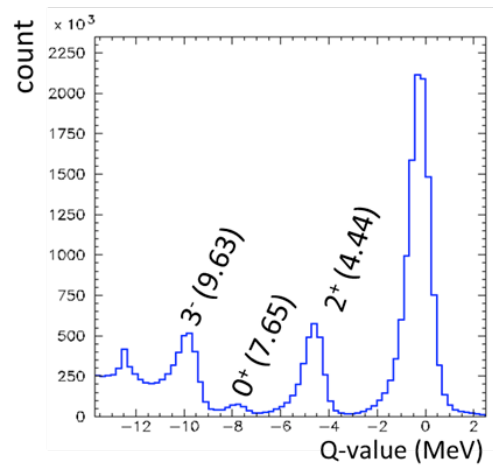

Fig. 2. Q-value spectrum collected at angles $\theta>30^{\circ}$ in the reaction $\mathrm{p}+{ }^{12} \mathrm{C}$ at $24 \mathrm{MeV}$.

\section{References}

1. F.Herwig S.M. Austin and J.C. Lattanzio Phys. Rev. C 73 (2006) 025802.

2. A. W. Obstt and W. J. Braithwaite phys.Rev.C 13 (1976) 2033

3. D.Chamberlin et al., Phys.Rev.C 9 (1974) 69

4. N. Davids et al Phys Rev C 11 (1975) 2063

5. H.-B. Mak et al Phys.Rev.C 12 (1975) 1158

6. M.Tsumura et al., Journal of Physics: Conference Series 569 (2014) 012051.

7. D. Chamberlin et al., Phys.Rev.C 10 (1974) 909

8. A.Pagano et al., Nucl.Phys. A 734 (2004) 504

9. R.G.Markham et al., Nucl.Phys. A270 (1976) 489

10. M.Freer and H.O.U.Fynbo, prog.part.and nucl. phys. 78(2014)1

11. https://www.Ins.infn.it/it/acceleratori/fribs-lns.html

12. L.Acosta et al., NIM A 715 (2013) 56

13. E. Pollacco et al., submitted to NIM

14. M.Alderighi et al NIM A 489 (2002) 257

15. F.Amorini et al IEEE trans. on Nucl. Sci. 1 (2012) 1772

16. R. Bassini et al., IEEE Trans. on Nucl. Sci. 1 (2006) 507

17. G.Cardella et al NIM A 799 (2015) 64 